\title{
Effects of Structured Resisted Exercises on Cognition Level Among Patients with Mild Cognitive Impairment
}

\author{
MUHAMMAD HASSAN ${ }^{1}$, SAJID RASHID ${ }^{2}$, REHAN RAMZAN KHAN ${ }^{3}$, MUHAMMAD USMAN KHALID ${ }^{4}$, HAROON \\ MANSHA ${ }^{5}$, HAYATULLAH KHALID ${ }^{6}$ \\ ${ }^{1,3-6}$ Assistant Professor, Multan College of Physiotherapy, Multan Medical \& Dental College, Multan, Pakistan. \\ ${ }^{2}$ Professor, Multan College of Physiotherapy, Multan Medical \& Dental College, Multan, Pakistan. \\ Correspondence to: Dr. Muhammad Hassan, MS-OMPT, Email: mhassansajjad91@gmail.com, Cell: 0321- 7352381
}

\begin{abstract}
Objective: To evaluate the effects of structured resistance exercises on cognition level among patients with mild cognitive impairment.

Methods: A quasi experimental trial was conducted on thirty patients with mild cognitive impairment $(\mathrm{MCl})$ from September 2020 to February 2021 at Ibn e Siena hospital, Multan. The total sample was randomly divided into two equal groups containing fifteen patients each; Group-A (Conventional pharmacological treatment) and GroupB (Resisted exercises). Group-A participants were treated with conventional pharmacological treatment cholinesterase inhibitors along with regular physical exercise while Group-B participants were treated with resistance exercises along with conventional pharmacological treatment. The standardized tools were used for data collection including Standardized mini mental state examination (SMMSE), Montreal cognitive assessment (MOCA), Trial making test A (TMT-A) and Trial making test B (TMT-B). Data was entered and analyzed by using SPSS 21.

Results: Independent samples T-test showed statistically significant difference after intervention for measures of cognitive performance. There was significant difference $(p<0.01)$ between pre and post intervention score of SMMSE $(20.60 \pm 1.75$ and $23.20 \pm 1.69)$ and MOCA $(17.60 \pm 1.35$ and $21.93 \pm 1.57)$. There was also significant difference between $(p<0.01)$ pre and post intervention score of TMT-A $(1.47 \pm 0.34 \& 1.23 \pm 0.04)$ and TMT-B $(2.51 \pm 0.04$ and $2.08 \pm 0.04)$.
\end{abstract}

Conclusion: Resistance exercises increases the cognitive levels of Mild Cognitive impairment patients.

Key Words: Cognitive dysfunction, Exercises, Dementia.

\section{INTRODUCTION}

The cognition is defined as the mental action or process of acquiring knowledge and understanding through thought, experience and the senses. Cognitive impairment affect older adults at age above fifty and it includes memory deficits, attentions deficits, and problem solving difficulties in activities of daily living. ${ }^{1}$ Cognitive impairment can affect learning something new, concentrating, remembering and decisions making. With older age decline of cognitive abilities begins to start. With advance decline of brain cells some persons encounter with memory problems, consciousness problems, judgement and reasoning issues and doing different tasks that can lead towards dementia. These functions are necessary to carry out basic activities of daily living. ${ }^{2}$

There are three types of cognitive impairments that are, mild, moderate and severe. Mild cognitive impairment also defines as it is early stage of dementia. Several studies shows that the prevalence of $\mathrm{MCl}$ in older adults is approximately 3- $42 \%{ }^{3}$ It stands between normal aging and dementia. It increases with age, at the age 60 years $1 \%$, at 70 years of age $12 \%$, at 80 years of age $30 \%$ and at 85 years of age $42 \%{ }^{4}$ Mild cognitive impairment is not progression of dementia but connect with memory that appears in progression of disease. ${ }^{5}$

Mild cognitive impairment is described by four criteria that include memory deficits, normal activities of daily living, executive functions and abnormal measure of cognition using education and age adjusted norms. ${ }^{6}$ The diagnostic criteria of $\mathrm{MCl}$ is made entirely on the basis of clinical background and on the decision of clinician. ${ }^{7}$ Recent diagnostic criteria for mild cognitive impairment include gradual decline of cognitive abilities for at least 6 months. ${ }^{5}$

There are several risk factors for $\mathrm{MCl}$ including hypertension, diabetes and obesity. ${ }^{8}$ Several studies on imaging of brain show that the main cause of $\mathrm{MCl}$ is shrinkage of hippocampus. There are several other causes which are associated with declination of cognition like different medications, bacterial or viral infections, deficiency of vitamins, depressive mood swings, smoking and use of alcohol. ${ }^{9}$

Physical activity has a protective effect on brain functions in older people. Although there are studies which provides evidence for efficacy cardiorespiratory and aerobics exercises for preventing cognitive decline in older people but very less is known about the effectiveness of structured resisted exercises in mild cognitive impairment. Therefore, this study was carried out to explore the effects of resistance training among patients with mild cognitive impairment and devising treatment plan inculcating resistance exercises upheld by evidence. The result of this study will be helpful for physical therapists engaged in care older population with cognitive impairment.

\section{METHODS}

A quasi experimental trial was conducted on thirty patients with mild cognitive impairment (MCl) from September 2020 to February 2021 at Ibn e Siena hospital and research institute, Multan after obtaining ethical approval. Patients having age of above 50 years with mild cognitive impairment confirmed by standardized mini mental scale examination having score of $18-23$ were included in the study. The Patients having scored greater than 25 and less 
than 18 in standardized mini mental scale examination, severe cognitive impairment due to brain tumours, TBI, stroke and Parkinson's disease were excluded. The patients were randomly divided into 2 equal groups by coin toss method containing fifteen patients each; Group-A (Conventional pharmacological treatment) and Group-B (Resisted exercises). The intervention protocol for Group-A (Conventional pharmacological treatment) participants was conventional pharmacological treatment that is cholinesterase inhibitors along with regular physical exercise whereas Group-B (Resisted exercises) was treated with resistance exercises for whole body by using thera bands and dumbbells ( 3 sets of 10 reps) along for the duration of fifty minutes along with conventional pharmacological treatment. The informed consent from all patients was taken before enrolment in the study.

Standardized outcome measure was used including standardized mini mental scale examination (SMMSE), trail making test-A (TMT-A), trail making Test-B (TMT-B) and Montreal cognitive assessment (MOCA). Patients were assessed twice; at baseline (Week 0) and at the termination of treatment (Week 6). The total duration of treatment consists of Six weeks with frequency of five sessions per week. (Total no. of sessions $=30$ ). End values of variables were calculated after completion of treatment session.

Data was analyzed by using SPSS version 21 . Frequency percentage, mean and standard deviation were used for presentation of categorical and demographic features. The level of significance was accepted as $\mathrm{P}<05$. Numeric variables were defined as mean \pm standard deviation.

\section{RESULTS}

The total participants in the study were 30 in numbers, divided in two groups. For control group, regular physical exercise along with conventional pharmacological treatment and for experimental group was resistance exercises for whole body and conventional pharmacological treatment. Among them 27 (90\%) were male and $3(10 \%)$ were females. The mean age of group $A$ and group $B$ was $53.86 \pm 3.4,54.40 \pm 4.76$ respectively. Mean SMMSE score was $19.93 \pm 1.62$ vs. $20.60 \pm 1.35$ at the start of treatment for Group-A (Pharmacological treatment) vs. Group-B (Resisted excises) respectively while it was $20.13 \pm 1.35$ vs. $23.20 \pm 1.35$ at the end of treatment for Group-A (Pharmacological treatment) vs. Group-B (Resisted excises) respectively. The mean score for MOCA was $17.93 \pm 1.66$ vs. $17.60 \pm 1.35$ at the start of treatment for Group-A (Pharmacological treatment) vs. Group-B (Resisted excises) respectively while it was $19.86 \pm 1.59$ vs. $21.93 \pm 1.57$ at the termination of treatment for Group-A (Pharmacological treatment) vs. Group-B (Resisted excises) respectively. The mean baseline score for TMT-A was $1.45 \pm 0.05$ vs. $1.47 \pm 0.03$ for Group-A (Pharmacological treatment) vs. Group-B (Resisted excises) respectively while post treatment score was 1.34 \pm 0.06 vs.1.23 0.04 for Group-A (Pharmacological treatment) vs. Group-B (Resisted excises) respectively. The mean baseline treatment score for TMT-B was 2.53 \pm

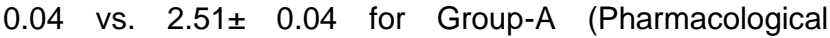
treatment) vs. Group-B (Resisted excises) respectively while post treatment score was $2.42 \pm 0.04$ vs. $2.08 \pm 0.04$ for Group-A (Pharmacological treatment) vs. Group-B (Resisted excises) respectively.

Table- I: SMMSE and MOCA Score in patients having cognitive dysfunction in Both Groups
\begin{tabular}{|l|l|l|l|}
\hline Mean Score & Mean \pm S.D. & p-value \\
\hline & Group-A (Pharmacological treatment) & $\begin{array}{l}\text { Group-B } \\
\text { (Resisted exercises) }\end{array}$ & \\
\hline & & $20.60 \pm 1.35$ & 0.23 \\
\hline Standardized Mini Mental State Examination (SMMSE) & $23.20 \pm 1.69$ & 0.00 \\
\hline Pre-treatment SMMSE (Week 0) & $19.93 \pm 1.62$ & $17.60 \pm 1.35$ & 0.55 \\
\hline Post- treatment SMMSE (Week 6) & $20.13 \pm 1.75$ & $21.93 \pm 1.57$ & 0.00 \\
\hline Montreal Cognitive Assessment (MOCA) & $17.93 \pm 1.66$ & $19.86 \pm 1.59$ & \\
\hline Pre-treatment MOCA (Week 0) & Post-treatment MOCA (Week 6)
\end{tabular}

Table-II: TMT-A \& TMT-B score in Patients having cognitive dysfunction in both groups

\begin{tabular}{l} 
Table-II: TMT-A \& TMT-B score in Patients having cognitive dysfunction in both groups \\
\begin{tabular}{|l|l|l|l|}
\hline Mean Score & Mean \pm S.D. & (Resisted & p-value \\
\hline & Group-A (Pharmacological treatment) & $\begin{array}{l}\text { Group-B } \\
\text { exercises) }\end{array}$ & \multicolumn{2}{l|}{} \\
\hline & & $1.47 \pm 0.03$ & 0.12 \\
\hline Trail Making Test-A (TMT-A) & $1.23 \pm 0.04$ & 0.00 \\
\hline Pre-treatment TMT-A (Week 0) & $1.45 \pm 0.05$ & $2.51 \pm 0.04$ & 0.35 \\
\hline Post-treatment TMT-A (Week 6) & $1.34 \pm 0.06$ & $2.08 \pm 0.04$ & 0.00 \\
\hline Trail Making Test-B (TMT-B) & $2.53 \pm 0.04$ & \\
\hline Pre-treatment TMT-B (Week 0) & $2.42 \pm 0.04$ &
\end{tabular} \\
\hline Post-treatment TMT-B (Week 6)
\end{tabular}

\section{DISCUSSION}

Resistance exercises are found to be more effective in patients with mild cognitive impairment in improving their cognitive functions including memory, recall and attention. There is also vital effect of resistance exercises on patients with dementia, ${ }^{10}$ and with history of fall and fall related factors in elderly people with $\mathrm{MCl} .{ }^{11}$ There is no particular treatment for mild cognitive impairment. Several pharmacological and non-pharmacological treatment plans are used to improve daily cognitive function and slow down the development rate of mild cognitive impairment and dementia. ${ }^{12}$

A study was done by SG Hong et al that concluded resistance exercises help the $\mathrm{MCl}$ patient to improve their cognitive function. They used MOCA as their cognitive measure tool that also showed significant improvement in post intervention measure. This study results showed significant difference in cognitive measure that include MOCA pre and post intervention ${ }^{13}$. Current study showed significant improvement in post treatment MOCA score and endorses the findings of this study. 
A study was conducted by Chia Liang and his colleagues to know about the results of acute aerobic and resistive exercises on muscle making cells improvement, and neurocognitive improvement in older adults having $\mathrm{MCl} .66$ patients were included in the study and they were equally divided into 3 groups. One group receives aerobic exercises, second group receives resistive exercises and the third group didn't receive any exercise. After repetitive trials it was observed that resistive exercises show great improvements in $\mathrm{MCl}$ patients. ${ }^{14}$ Our study also showed improvement in $\mathrm{MCl}$ patients being treated with resisted exercises in addition to conventional pharmacological treatment. So the findings of current study are in agreement with the available literature.

W.C. LiYangLei conducted systematic review to check the cognitive and psychological improvement in people having $\mathrm{MCl}$. It was observed that physical modalities used in these conditions have no effect but the exercise program show remarkable results. After number of repetitions, it was observed that aerobic exercises have moderate effect on $\mathrm{MCl}$ patients whereas resistance training exercises has remarkable effect in improving cognition and psychological factors ${ }^{15}$. The current study also showed similar improvement in cognition of $\mathrm{MCl}$ patients.

$\mathrm{L}$ Liang et al conducted a study and concluded that momentum based dumbbell exercises have potential benefit for improving cognitive functional abilities in older adults with mild cognitive impairment. They used trail making test B (TMT-B) as their assessment tool that showed significant improvement in post intervention measures. In current study trail making test B (TMT-B) also showed significant difference in pre and post treatment measures. ${ }^{16}$

A randomized control trial conducted Tsai C-Land his colleagues to investigate the effects of 12-week resistance exercise program along with an elastic band on EEG patterns and cognitive function in older $\mathrm{MCl}$ patients. $\mathrm{MCl}$ group received resistive exercises whereas control group didn't receive any physical therapy protocol. At the end of the session it was observed that this exercise program improves bodily fitness of an elder people having $\mathrm{MCl}$. It was concluded that resisted exercise plan can be the effective treatment protocol for the cognitive decline and improving fitness. ${ }^{17}$ The results of our study showed similar improvement in cognition after addition of resisted exercises to conventional pharmacological treatment.

\section{CONCLUSION}

Resistance exercises are significant in improving cognition level among patients with mild cognitive impairment.

\section{REFERENCES}

1. Pawlik K and d'Ydewalle G. Psychological concepts: An international historical perspective. Psychology Press, 2020.

2. Huckans $M$, Hutson L, Twamley $E$, Jak $A$, Kaye $J$ and Storzbach D. Efficacy of cognitive rehabilitation therapies for mild cognitive impairment $(\mathrm{MCl})$ in older adults: working toward a theoretical model and evidence-based interventions. Neuropsychology review. 2013; 23: 63-80.

3. Foran A, Mathias $\mathrm{J}$ and Bowden S. Effectiveness of sorting tests for detecting cognitive decline in older adults with dementia and other common neurodegenerative disorders: a meta-analysis. Neuroscience \& Biobehavioral Reviews. 2020.

4. Power MC, Mormino E, Soldan A, et al. Combined neuropathological pathways account for age-related risk of dementia. Annals of neurology. 2018; 84: 10-22.

5. Nuzum H, Stickel A, Corona M, Zeller M, Melrose RJ and Wilkins SS. Potential benefits of physical activity in $\mathrm{MCl}$ and dementia. Behavioural neurology. 2020; 2020.

6. Anderson ND. State of the science on mild cognitive impairment (MCI). CNS spectrums. 2019; 24: 78-87.

7. Edmonds EC, McDonald CR, Marshall A, et al. Early versus late $\mathrm{MCl}$ : Improved $\mathrm{MCl}$ staging using a neuropsychological approach. Alzheimer's \& Dementia. 2019; 15: 699-708.

8. Wang $F$, Zhao M, Han Z, et al. Long-term subclinical hyperglycemia and hypoglycemia as independent risk factors for mild cognitive impairment in elderly people. The Tohoku journal of experimental medicine. 2017; 242: 121-8.

9. Keyimu K, Zhou X-H, Miao H-J and Zou T. Mild cognitive impairment risk factor survey of the Xinjiang Uyghur and Han elderly. International journal of clinical and experimental medicine. 2015; 8: 13891.

10. Öhman H, Savikko N, Strandberg TE and Pitkälä KH. Effect of physical exercise on cognitive performance in older adults with mild cognitive impairment or dementia: a systematic review. Dementia and geriatric cognitive disorders. 2014; 38: 347-65.

11. Song D, Doris S, Li PW and Lei Y. The effectiveness of physical exercise on cognitive and psychological outcomes in individuals with mild cognitive impairment: A systematic review and meta-analysis. International journal of nursing studies. 2018; 79: 155-64.

12. Chertkow H, Massoud F, Nasreddine Z, et al. Diagnosis and treatment of dementia: 3 . Mild cognitive impairment and cognitive impairment without dementia. Canadian Medical Association Journal. 2008; 178: 1273-85.

13. Hong S-G, Kim J-H and Jun T-W. Effects of 12-week resistance exercise on electroencephalogram patterns and cognitive function in the elderly with mild cognitive impairment: a randomized controlled trial. Clin J Sport Med. 2017.

14. Chia-LiangTsaiaJozefUkropecbBarbaraUkropcovábcd1MingChyiPaief1. An acute bout of aerobic or strength exercise specifically modifies circulating exerkine levels and neurocognitive functions in elderly individuals with mild cognitive impairment. 2018, p. 272-84.

15. W.C.LiYangLei DSFY. The effectiveness of physical exercise on cognitive and psychological outcomes in individuals with mild cognitive impairment: A systematic review and meta-analysis. March 2018, p. 155-64.

16. Lü J, Sun M, Liang L, Feng $Y$, Pan $X$ and Liu Y. Effects of momentum-based dumbbell training on cognitive function in older adults with mild cognitive impairment: a pilot randomized controlled trial. Clinical interventions in aging. 2016; $11: 9$.

17. Tsai C-L, Ukropec J, Ukropcová B and Pai M-C. An acute bout of aerobic or strength exercise specifically modifies circulating exerkine levels and neurocognitive functions in elderly individuals with mild cognitive impairment. Neurolmage: Clinical. 2018; 17: 272-84. 